\title{
Clinical Efficacy of Docetaxel Combined with Cisplatin in the Treatment of Laryngeal Tumors and Nursing Intervention
}

\author{
ZIYAN CAI, YAN SUN*AND LEI CAO \\ Department of Otolaryngology, Zhangjiagang Traditional Chinese Medicine Hospital Affiliated to Nanjing University of Chinese \\ Medicine, Zhangjiagang, Jiangsu 215600, China
}

Cai et al.: Combined Efficacy of Docetaxel and Cisplatin in the Treatment of Laryngeal Tumors

\begin{abstract}
To explore the effects of docetaxel combined with cisplatin in the treatment of laryngeal tumors and nursing intervention. A total of 60 laryngeal tumor patients admitted from January 2015 to January 2021 were selected and divided into a control group and an observation group according to the random number table method. The patients in the control group received intravenous drip of cisplatin injection and fluorouracil injection whereas observation group received cisplatin injection and docetaxel injection. At the same time, patients in the control group received routine nursing intervention whereas observation group received comprehensive nursing intervention. The short-term efficacy, toxic and side effects, 1-3 y survival rate, thyroid function and tumor cell apoptosis were compared and analyzed between the two groups. The results showed that the total effective rate of treatment in the observation group was as high as $\mathbf{8 6 . 6 7 \%}$, which was significantly higher than the $63.34 \%$ in the control group. The difference was statistically significant $(p<0.05)$. The incidence of severe toxic and side effects in the observation group was lower than that in the control group, but the difference was not statistically significant $(p>0.05)$. The $1 \mathrm{y}$ and 3 y survival rates in the observation group were $86.67 \%$ and $60.00 \%$, which were significantly higher than the $66.67 \%$ and $43.33 \%$ in the control group. The difference was statistically significant $(\mathbf{p}<\mathbf{0 . 0 5})$. After treatment, the thyroid stimulating hormone level in the observation group was significantly higher than that in the control group $(p<0.05)$ and triiodothyronine and tetraiodothyronine levels in the observation group were significantly lower than those in the control group $(\mathbf{p}<\mathbf{0 . 0 5})$; the apoptosis rate of tumor cells in the observation group was significantly higher than that in the control group $[(29.32 \pm 2.63) \%$ vs. $(20.97 \pm 1.69) \%]$. Docetaxel combined with cisplatin simultaneous radiotherapy has a better clinical effect on laryngeal tumors and a higher survival rate, which is worthy of clinical promotion.
\end{abstract}

Key words: Laryngeal tumor, cisplatin, docetaxel, nursing intervention

Laryngeal malignant tumors are a type of malignant tumors that occur in the supraglottic area, the glottal area and the subglottic area. Laryngeal tumors mostly refer to laryngeal squamous cell carcinoma, accounting for most of its pathological types and occurring in men of $50 \mathrm{y}-70 \mathrm{y}$, which is one of the most common malignant tumors of the head and neck and also the second most common cancer of the respiratory tract after lung cancer. The incidence of laryngeal tumors in China is increasing year by year ${ }^{[1,2]}$. The main treatment method for laryngeal tumors is surgical resection, combined with radiotherapy, chemotherapy and other comprehensive treatments. However, surgical treatment can easily destroy the patient's normal laryngeal structure and severely affect the patient's vocal function. Therefore, the use of radiotherapy and drug

*Address for correspondence

E-mail: yangrh169@163.com therapy has great advantages in preserving the patient's normal vocal function and laryngeal structure. Hence radiotherapy combined with chemotherapy is generally the first choice in clinical practice ${ }^{[3]}$. Cisplatin, similar to a bifunctional alkylating agent and belonging to the cell cycle non-specific drug, with strong cytotoxicity, can inhibit the Deoxyribonucleic Acid (DNA) replication of cancer cells and can damage the structure of the cell membrane. It is reported that the total effective rate of laryngeal malignant tumor patients receiving cisplatin combined with fluorouracil is more than $45 \%$. Cisplatin combined with fluorouracil can also increase the sensitivity of radiotherapy, but there are only a few related reports. Therefore, this study aimed to explore the effect of docetaxel combined with cisplatin in the treatment of laryngeal tumors and nursing intervention. 


\section{MATERIALS AND METHODS}

\section{General data:}

A total of 60 laryngeal tumor patients admitted from January 2015 to January 2021 were selected and divided into a control group and an observation group according to the random number table method with 30 patients in each group. The study was approved by the ethics committee of our hospital and all patients and their families were informed of the study contents and signed the consent. Among them, there were 17 males and 13 females in the control group, aged $51 \mathrm{y}-77 \mathrm{y}$ old, with an average age of $62.3 \pm 5.8 \mathrm{y}$; there were 15 males and 15 females in the observation group, aged 50 $\mathrm{y}-78 \mathrm{y}$ old, with an average age of $62.6 \pm 5.5 \mathrm{y}$. Through statistical analysis, there was no statistically significant difference in general data between the two groups $(p>0.05)$ and they were comparable.

\section{Treatment methods:}

The patients in the control group received intravenous drip of cisplatin injection (Yunnan Gejiu Biopharmaceutical Co., Ltd.) and fluorouracil injection (Tianjin Jinyao Pharmaceutical Co., Ltd.). On 1-3 d, the dosage of cisplatin injection was $25 \mathrm{mg} / \mathrm{m}^{2}$; On 1 to $5 \mathrm{~d}$, the dosage of fluorouracil injection was 500 $\mathrm{mg} / \mathrm{m}^{2}$. The patients in the observation group received intravenous drip of cisplatin injection and docetaxel injection (Jiangsu Hengrui Pharmaceutical Co., Ltd.). The dosage of cisplatin injection was the same as that in the control group and the dosage of docetaxel injection was $75 \mathrm{mg} / \mathrm{m}^{2}$. Both groups of patients took $21 \mathrm{~d}$ as a treatment cycle and a total of 2 cycles of treatment were carried out.

\section{Nursing intervention:}

The patients in the control group received routine nursing intervention, i.e., they explained relevant knowledge to patients before radiotherapy and chemotherapy, so that they can understand the preparatory preparation, process, efficacy, toxicity and side effects of radiotherapy and chemotherapy, promptly deal with uncomfortable reactions during radiotherapy and chemotherapy and remind patients to pay attention to diet, etc., ${ }^{[4]}$. On the basis of routine nursing, the patients in the observation group received comprehensive nursing intervention for the toxic and side effects of radiotherapy and chemotherapy on the gastrointestinal tract, skin and oral mucosa, mainly including the following aspects.

\section{Psychological nursing: Chemotherapy and}

radiotherapy caused a lot of physical discomfort for patients and they were easily nervous and anxious. Therefore, during chemotherapy and radiotherapy, the number of visits to the ward was increased and the patients were communicated more to understand their specific illness, mental state, personality characteristics, family status, income and expenditure situation, etc., to disperse fears. The patient's trust and sense of practicality in medical staff were increased and the patient's psychological needs were satisfied ${ }^{[5]}$.

Dietary guidance: During radiotherapy and chemotherapy, in order to prevent dry mouth and tongue, patients were promptly supplied with adequate water and a light diet. The easy-to-digest foods that were soft, with less fiber and rich in calories, protein and vitamins were chosen. Patients with no appetite ate smaller meals. In order to avoid damage to the oral mucosa, patients were fasted hard for fried, rough, hot, prickly and spicy foods ${ }^{[6]}$.

Nursing intervention for gastrointestinal reactions: During chemotherapy, patients often had gastrointestinal reactions such as abdominal pain, diarrhea, nausea and vomiting. During nursing intervention, appropriate anti-emetic treatment was given and the patients were taught to self-stop vomiting easily and intentionally and provided with favorite light music to ease the patient's excessive tension and reduce the gastrointestinal reaction. The frequency of vomiting was recorded, the nature, color and amount of the vomiting contents were observed and the patients were replenished with fluids and electrolytes in time ${ }^{[7]}$.

Nursing intervention for oral mucosal reaction: Regarding the nursing for oral mucosa, patients were advised to pay attention to oral hygiene, use a soft toothbrush to brush their teeth carefully in the morning, evening and after eating and use Kangfuxin liquid to gargle. If there was a false membrane in the oral cavity, $1.5 \%$ hydrogen peroxide solution was used to gargle. If the patient was prone to dry mouth, plenty of water was drunk or appropriate amount of acidic food was taken to stimulate saliva secretion. Smoking and drinking were quit and treatment for oral inflammation was actively carried out. For patients with strong pain, they rinsed the mouth with a multi-vitamin mouthwash containing local anesthesia before meals ${ }^{[8]}$.

Nursing intervention for skin reactions: Before and during radiotherapy, the patient's skin nursing was emphasized. Before radiotherapy, patients were told to have a haircut and shave to avoid skin abrasions during radiotherapy. Patients wore loose, soft and hygroscopic 
cotton underwear when undergoing chemotherapy. The skin of the irradiated field was kept clean and dry. Warm water was used when washing. Irritating detergents such as soap or alcohol could not be used. Irritating ointment was not applied to the skin of the irradiated field and medicine containing vitamin B12 was applied locally and adhesive tape was not pasted. If the skin was itchy, it could not be scratched and could be patted locally. The skin was not tore for desquamation, patients were diverted attention or relaxed to reduce itching ${ }^{[9]}$.

\section{Observation indexes:}

The short-term curative effect was evaluated 2 mo after the radiotherapy, using the Response Evaluation Criteria In Solid Tumors (RECIST). Complete Remission (CR) is the visible tumor disappeared completely for more than $4 \mathrm{w}$; Partial Response (PR) is the product of the maximum diameter or the maximum vertical diameter of the lesion decreased by $50 \%$ for more than $4 \mathrm{w}$; Stable Disease (SD) is the product of the two diameters decreased by less than $50 \%$ or increased by $<25 \%$ for more than $4 \mathrm{w}$; Progressive Disease (PD) is the product of the two diameters increased by more than $25 \%$;

\section{Response Rate $(\mathrm{RR})=\mathrm{CR}+\mathrm{PR}$}

The incidence of severe toxic and side effects of the two groups of patients, according to the Radiation Therapy Oncology Group (RTOG) acute radiation injury classification standard and the World Health Organization (WHO) anti-cancer drug acute and subacute toxicity classification standard, $\geq$ III was considered as severe toxic and side effects. The follow-up ended in June 2021 and the patients were followed up for 24 mo-48 mo to calculate the survival rate of the patients. Statistics of the three indicators of Thyroid Stimulating Hormone (TSH), serum total Triiodothyronine (T3) and serum total Tetraiodothyronine (T4) before and after treatment in the two groups of patients ${ }^{[10]}$.

Tumor cell apoptosis rate: The apoptosis rate of tumor cells in the two groups of laryngeal tumor patients after treatment was observed and compared. The apoptosis rate of tumor cells in patients was detected by Terminal Deoxynucleotidyl Transferase dUTP Nick End Labeling (TUNEL) method: Wash once with Hank's Balanced Salt Solution (HBSS) or Phosphate Buffered Saline (PBS); If the cells were not adhered firmly, the sample was dried to make the cells adhere firmly; Immunostaining fixative (P0098) or $4 \%$ paraformaldehyde was used to fix the cells for about $1 \mathrm{~h}$; Wash the cells again with HBSS or PBS; PBS with a concentration of $0.1 \%$ Triton X-100 was added for incubation in an ice bath for $2 \min ^{[11]}$. Calculation formula is mentioned below:

Tumor cell apoptosis rate $=1$-(Optical Density (OD) value after treatment-OD value before treatment $) \times 100 \%$

European Organization for the Research and Treatment of Cancer Quality of Life Questionnaire (EORTC QLQ-C30): Questionnaire was a general questionnaire used to assess the quality of life of cancer patients. It was often applied to patients with head and neck tumors. It included 30 items and 5 functional areas (physical, role, cognitive, emotional and social function). The higher the score in the functional area, better the functional status and quality of life. Higher the score in the symptom area, worse the quality of life (more the symptoms or problems).

\section{Statistical analysis:}

Statistical Package for the Social Sciences (SPSS) 21.0 software was used to statistically analyze the data of this study. The $t$ test was used to compare the measurement data and the chi-square test was used to compare the count data. The results were statistically significant when $\mathrm{p}<0.05$.

\section{RESULTS AND DISCUSSION}

The total effective rate of treatment in the observation group was as high as $86.67 \%$, which was significantly higher than the $63.34 \%$ in the control group. The difference was statistically significant $(p<0.05)$, as shown in Table 1. The incidence of severe toxic and side effects in the observation group was lower than that in the control group, but the difference was not statistically significant ( $\mathrm{p}>0.05)$, as shown in Table 2 . The $1 \mathrm{y}$ and $3 \mathrm{y}$ survival rates in the observation group were $86.67 \%$ and $60.00 \%$, which were significantly higher than the $66.67 \%$ and $43.33 \%$ in the control group. The difference was statistically significant, as shown in Table 3.

After treatment, the TSH level in the observation group was significantly higher than that in the control group $(p<0.05)$ and the T3 and T4 levels in the observation group were significantly lower than those of in control group ( $p<0.05)$, as shown in Table 4 . The apoptosis rate of tumor cells in the observation group was significantly higher than that in the control group $[(29.32 \pm 2.63)$ $\%$ vs. $(20.97 \pm 1.69) \%, \mathrm{p}<0.05]$, as shown in Table 5 . After treatment, the EORTC QLQ-C30 scores in the observation group were significantly higher than those in the control group $(\mathrm{p}<0.05)$, as shown in Table 6 . This study showed that after comprehensive nursing, the 
TABLE 1: SHORT-TERM EFFICACY OF THE TWO GROUPS OF PATIENTS

\begin{tabular}{|c|c|c|c|c|c|c|}
\hline Group & $\mathrm{n}$ & CR & PR & SD & PD & $\begin{array}{c}\text { Total effective } \\
\text { rate }\end{array}$ \\
\hline $\begin{array}{l}\text { Observation } \\
\text { group }\end{array}$ & 30 & $9(30.00)$ & $17(56.67)$ & $3(10.00)$ & $1(3.33)$ & 86.67 \\
\hline Control group & 30 & $5(16.67)$ & $14(46.67)$ & $7(23.33)$ & $4(13.33)$ & 63.34 \\
\hline$x^{2}$ & \multicolumn{6}{|c|}{12.367} \\
\hline $\mathrm{p}$ & \multicolumn{6}{|c|}{$<0.05$} \\
\hline
\end{tabular}

TABLE 2: THE INCIDENCE OF SEVERE TOXIC AND SIDE EFFECTS OF THE TWO GROUPS OF PATIENTS

\begin{tabular}{|c|c|c|c|c|c|}
\hline Group & $\mathrm{n}$ & Leukopenia & $\begin{array}{c}\text { Nausea and } \\
\text { vomiting }\end{array}$ & Thrombocytopenia & Stomatitis \\
\hline Observation group & 30 & $13(43.33)$ & $5(16.67)$ & $2(6.67)$ & $2(6.67)$ \\
\hline Control group & 30 & $12(40.00)$ & $3(10.00)$ & $3(10.00)$ & $3(10.00)$ \\
\hline$x^{2}$ & & & 1.087 & & \\
\hline $\mathrm{p}$ & & & $>0.05$ & & \\
\hline
\end{tabular}

TABLE 3: 1 Y AND 3 Y SURVIVAL RATES OF THE TWO GROUPS OF THE PATIENTS

\begin{tabular}{lccc}
\hline Group & $\mathbf{n}$ & 1 y survival rate & 3 y survival rate \\
\hline Observation group & 30 & $26(86.67)$ & $18(60.00)$ \\
Control group & 30 & $20(66.67)$ & $13(43.33)$ \\
$x^{2}$ & & 1.032 & $<0.05$ \\
$p$ & & & \\
\hline
\end{tabular}

TABLE 4: CHANGES IN THYROID FUNCTION BEFORE AND AFTER TREATMENT

\begin{tabular}{lcccc}
\hline \multirow{2}{*}{ Indicator } & \multicolumn{2}{c}{ Control group $(\mathrm{n}=30)$} & \multicolumn{2}{c}{ Observation group $(\mathrm{n}=30)$} \\
\cline { 2 - 5 } & Before treatment & After treatment & Before treatment & After treatment \\
\hline TSH $(\mathrm{nmol} / \mathrm{l})$ & $1.29 \pm 0.67$ & $1.79 \pm 0.48$ & $1.31 \pm 0.59$ & $2.61 \pm 0.61^{*}$ \\
T3 $(\mathrm{nmol} / \mathrm{l})$ & $6.01 \pm 0.39$ & $5.46 \pm 0.52$ & $6.11 \pm 0.41$ & $4.09 \pm 0.44^{*}$ \\
$\mathrm{~T} 4(\mu \mathrm{lU} / \mathrm{l})$ & $19.17 \pm 2.62$ & $17.68 \pm 2.15$ & $19.23 \pm 2.57$ & $16.17 \pm 2.24^{*}$ \\
\hline
\end{tabular}

Note: Observation group vs. control group after treatment, ${ }^{*} \mathrm{p}<0.05$

TABLE 5: THE APOPTOSIS RATE OF TUMOR CELLS OF THE TWO GROUPS OF PATIENTS

\begin{tabular}{lcc}
\hline Group & Before treatment & After treatment \\
\hline Observation group & $7.46 \pm 1.48$ & $29.32 \pm 2.63^{*}$ \\
Control group & $7.52 \pm 1.19$ & $20.97 \pm 1.69$ \\
\hline
\end{tabular}

Note: Observation group vs. control group after treatment, ${ }^{*} \mathrm{p}<0.05$

TABLE 6: EORTC QLQ-C30 SCORES OF TWO GROUPS OF PATIENTS 6 MO AFTER OPERATION

\begin{tabular}{lcccc}
\hline Item & Observation group & Control group & $\mathrm{t}$ & $\mathrm{P}$ \\
\hline Physical function & $63.03 \pm 21.34$ & $51.23 \pm 13.96$ & 2.534 & 0.014 \\
Role function & $50.57 \pm 12.21$ & $42.67 \pm 13.09$ & 2.417 & 0.019 \\
Emotional function & $55.50 \pm 15.75$ & $40.23 \pm 12.76$ & 4.152 & 0.014 \\
Cognitive function & $66.30 \pm 12.73$ & $56.77 \pm 13.02$ & 2.894 & 0.006 \\
Social function & $43.80 \pm 13.44$ & $37.43 \pm 14.12$ & 2.971 & 0.005 \\
\hline
\end{tabular}


patient's quality of life has been significantly improved.

Laryngeal tumor is a tumor that originates from the patient's laryngeal mucosal tissues.

The cell types of most laryngeal tumor patients are mainly squamous cells clinically and they have a very high incidence rate in clinical practice in China. The clinical causes of the disease are relatively complicated. Related studies have shown that laryngeal tumor is closely related to the work, living environment and personal eating habits, such as excessive drinking, smoking, chronic laryngitis and inflammatory stimuli or viral infections, air pollution and sex hormones etc. Among all the risk factors that cause patients to develop the disease, smoking is one of the highest risk factors for onset of laryngeal tumor patients ${ }^{[12,13]}$. Most laryngeal tumor patients are diagnosed as early laryngeal cancer when they are admitted to the hospital for examination. The main clinical symptoms are sore throat, hoarseness, blood in the sputum and obvious foreign body sensation in the throat. If effective and timely treatment is not given to the patients in time, it will cause a very serious threat to the patient's daily life and health ${ }^{[14]}$.

At present, the treatment of laryngeal tumors uses platinum-based simultaneous radiotherapy and chemotherapy. Paclitaxel, docetaxel, 5-fluorouracil and platinum-based combined simultaneous radiotherapy are currently used common treatment options. The platinum type in the classic chemotherapy regimen is cisplatin. However, due to the high incidence of gastrointestinal reactions, the treatment time is often prolonged and interrupted, thereby reducing the effective rate. Docetaxel is a new type of anti-cancer drug. Its anti-cancer mechanism is similar to that of cisplatin i.e., it binds to the DNA bases of cancer cells to hinder DNA replication, thereby exerting its antitumor effect, broad-spectrum anti-cancer activity. It has a lower response to gastrointestinal toxicity and no cross-resistance to other platinum drugs, which can be used to treat head and neck cancer, small cell lung cancer, non-small cell lung cancer, esophageal cancer, bladder cancer, testicular cancer, ovarian cancer, etc. Docetaxel can be used alone or concurrently with other chemotherapy drugs, molecular targeted drugs and radiotherapy and it has a sensitizing effect on radiotherapy. This study compared the effects of docetaxel combined with cisplatin on laryngeal tumors and nursing intervention.

This study showed that the total effective rate of treatment in the observation group was significantly higher than that in the control group and the difference was statistically significant $(\mathrm{p}<0.05)$. The $1 \mathrm{y}$ and $3 \mathrm{y}$ survival rate in the observation group was significantly higher than that in the control group, suggesting that the effect of docetaxel combined with cisplatin in the treatment of concurrent radiotherapy for laryngeal tumors was definite, better than that of fluorouracil combined with cisplatin, which was consistent with the results of existing studies ${ }^{[15,16]}$. With the development and maturity of pharmacology, chemotherapeutic drugs have been continuously optimized. Docetaxel is a semi-synthetic paclitaxel drug with good intracellular solubility and can bind to free tubulin to enhance the stability of microtubules, reduce the amount of tubulin and inhibit tumor division. Some data have showed that the combined application of docetaxel and radiotherapy can exert a synergistic effect, which can promote the synchronization of tumor cell cycle, promote cell retention in the Second Growth Phase/Mitotic Phase ( $\mathrm{G} 2 / \mathrm{M}$ phase) and enhance the killing effect of radiation on tumor cells. At the same time, the results of the study showed that there was no significant difference in the incidence of toxic and side effects between the two groups of patients, suggesting that both two treatment options have high safety, toxicity and side effects are within the tolerable range of patients.

The results of this study indicated that the TSH level in the observation group was significantly higher than that in the control group $(\mathrm{p}<0.05)$; the T3 and T4 levels in the observation group were significantly lower than those in the control group $(p<0.05)$. In anatomy, the position of the human larynx was closer to the thyroid gland. When patients undergo related anti-cancer treatments, there will be varying degrees of hypothyroidism, especially when patients undergo partial laryngectomy, part of the thyroid gland is removed at the same time and the function of hypothyroidism is significantly increased. In the clinical treatment of laryngeal tumors, it is necessary to strictly control the indicators of thyroid tissues, especially for laryngeal cancer patients at advanced stages, subglottic patients or patients with significant thyroid palpable nodules. Hypothyroidism is the most common complication clinically. The main clinical manifestation is the decrease of T3 and T4 levels and the increase of TSH level in the patient's serum ${ }^{[17]}$. Therefore, the results of this study indicated that the application of docetaxel combined with cisplatin in the clinical treatment of laryngeal cancer patients can better protect thyroid function and its efficacy is significant.

Functional areas are mainly manifested as physiological 
functions (activity, diet, rest, daily self-care, etc.), emotions, communication and social activities, appearance changes, energy, family relations, etc. Damage to the physiological function of laryngeal cancer patients after chemotherapy is the primary factor that affects their quality of life. After chemotherapy, the integrity of the respiratory tract is damaged and the physiological barrier of breathing is lost, which changes the path of the respiratory tract, causing adverse reactions such as shortness of breath and dysphagia to directly reduce the quality of life, making it difficult for patients to return to normal life and seriously affecting social functions ${ }^{[18,19]}$. The results of this study showed that all functional areas of the patient were greatly affected after treatment, changing the original life state and producing bad emotions (such as tension, anxiety, irritability, depression, etc.), which seriously affected the patient's sleep quality and nutrition situation. The results of this study showed that the functional area scores of patients in the observation group were higher than those in the control group $(\mathrm{p}<0.05)$, indicating that by training patients and their family members to master new knowledge and skills in maintaining throats, nurses acted as the person responsible for nursing, met the medical needs of patients with the least resources, made the patients transition from passively accepting the care of others to self-care, enhanced the effectiveness of self-care and achieved the purpose of improving the quality of life after surgery.

In conclusion, docetaxel combined with cisplatin simultaneous radiotherapy has a better clinical effect on laryngeal tumors, with a higher survival rate, which is worthy of clinical promotion.

\section{Acknowledgements:}

This work was supported by the Zhangjiagang Traditional Chinese Medicine Hospital.

\section{Conflict of interests:}

The authors declared no conflicts of interest.

\section{REFERENCES}

1. Paccagnella A, Orlando A, Marchiori C, Zorat PL, Cavaniglia $\mathrm{G}$, Sileni VC, et al. Phase III trial of initial chemotherapy in stage III or IV head and neck cancers: A study by the Gruppo di Studio sui Tumori della Testa e del Collo. J Natl Cancer Inst 1994;86(4):265-72.

2. Domenge C, Hill C, Lefebvre JL, de Raucourt D, Rhein B, Wibault $\mathrm{P}$, et al. Randomized trial of neoadjuvant chemotherapy in oropharyngeal carcinoma. Br J Cancer 2000;83(12):1594-8.

3. Zorat PL, Paccagnella A, Cavaniglia G, Loreggian L, Gava A, Mione CA, et al. Randomized phase III trial of neoadjuvant chemotherapy in head and neck cancer: 10-year follow-up. J Natl Cancer Inst 2004;96(22):1714-7.
4. Bonner JA, Harari PM, Giralt J, Azarnia N, Shin DM, Cohen $\mathrm{RB}$, et al. Radiotherapy plus cetuximab for squamouscell carcinoma of the head and neck. New Eng J Med 2006;354(6):567-78.

5. Vermorken JB, Remenar E, Van Herpen C, Gorlia T, Mesia $\mathrm{R}$, Degardin $\mathrm{M}$, et al. Cisplatin, fluorouracil and docetaxel in unresectable head and neck cancer. New Eng J Med 2007;357(17):1695-704.

6. Posner MR, Hershock DM, Blajman CR, Mickiewicz E, Winquist E, Gorbounova V, et al. Cisplatin and fluorouracil alone or with docetaxel in head and neck cancer. New Eng $\mathrm{J}$ Med 2007;357(17):1705-15.

7. Pointreau Y, Garaud P, Chapet S, Sire C, Tuchais C, Tortochaux $\mathrm{J}$, et al. Randomized trial of induction chemotherapy with cisplatin and 5-fluorouracil with or without docetaxel for larynx preservation. J Natl Cancer Inst 2009;101(7):498-506.

8. Pignon JP, Le Maitre A, Maillard E, Bourhis J, Mach-Nc Collaborative Group. Meta-analysis of chemotherapy in head and neck cancer (MACH-NC): An update on 93 randomised trials and 17,346 patients. Radiother Oncol 2009;92(1):4-14.

9. Paccagnella A, Ghi MG, Loreggian L, Buffoli A, Koussis H, Mione CA, et al. Concomitant chemoradiotherapy versus induction docetaxel, cisplatin and 5 fluorouracil (TPF) followed by concomitant chemoradiotherapy in locally advanced head and neck cancer: A phase II randomized study. Ann Oncol 2010;21(7):1515-22.

10. Zenda S, Matsuura K, Tachibana H,HommaA, Kirita T, Monden $\mathrm{N}$, et al. Multicenter phase II study of an opioid-based pain control program for head and neck cancer patients receiving chemoradiotherapy. Radiother Oncol 2011;101(3):410-4.

11. Haddad R, O'Neill A, Rabinowits G, Tishler R, Khuri F, Adkins $\mathrm{D}$, et al. Induction chemotherapy followed by concurrent chemoradiotherapy (sequential chemoradiotherapy) $v s$. concurrent chemoradiotherapy alone in locally advanced head and neck cancer (PARADIGM): A randomised phase 3 trial. Lancet Oncol 2013;14(3):257-64.

12. Hitt R, Grau JJ, Lopez-Pousa A, Berrocal A, García-Girón $\mathrm{C}$, Irigoyen $\mathrm{A}$, et al. A randomized phase III trial comparing induction chemotherapy followed by chemoradiotherapy versus chemoradiotherapy alone as treatment of unresectable head and neck cancer. Ann Oncol 2014;25(1):216-25.

13. Cohen EE, Karrison TG, Kocherginsky M, Mueller J, Egan $\mathrm{R}$, Huang $\mathrm{CH}$, et al. Phase III randomized trial of induction chemotherapy in patients with N2 or N3 locally advanced head and neck cancer. J Clin Oncol 2014;32(25):2735.

14. Guigay J, Fayette J, Dillies AF, Sire C, Kerger JN, Tennevet I, et al. Cetuximab, docetaxel and cisplatin as first-line treatment in patients with recurrent or metastatic head and neck squamous cell carcinoma: A multicenter, phase II GORTEC study. Ann Oncol 2015;26(9):1941-7.

15. Smith TJ, Bohlke K, Lyman GH, Carson KR, Crawford J, Cross SJ, et al. Recommendations for the use of WBC growth factors: American Society of Clinical Oncology clinical practice guideline update. J Clin Oncol 2015;33(28):3199-212.

16. Specenier PM, Remenar E, Buter J, Schrijvers DL, Bergamini C, Licitra LF, et al. TPF plus cetuximab induction chemotherapy followed by biochemoradiation with weekly cetuximab plus weekly cisplatin or carboplatin: A randomized phase II EORTC trial. Ann Oncol 2017;28(9):2219-24.

17. Kim R, Hahn S, Shin J, Ock CY, Kim M, Keam B, et al. The effect of induction chemotherapy using docetaxel, cisplatin and fluorouracil on survival in locally advanced head and neck squamous cell carcinoma: A meta-analysis. Cancer Res Treat 2016;48(3):907-16. 
18. Yokota $\mathrm{T}$, Tachibana H, Konishi $\mathrm{T}$, Yurikusa $\mathrm{T}$, Hamauchi $\mathrm{S}$, Sakai K, et al. Multicenter phase II study of an oral care program for patients with head and neck cancer receiving chemoradiotherapy. Support Care Cancer 2016;24(7):3029-36.

19. Tanaka Y, Yoshida K, Tanahashi T, Okumura N, Matsuhashi $\mathrm{N}$, Yamaguchi K. Phase II trial of neoadjuvant chemotherapy with docetaxel, nedaplatin and S1 for advanced esophageal squamous cell carcinoma. Cancer Sci 2016;107(6):764-72.
This is an open access article distributed under the terms of the Creative Commons Attribution-NonCommercial-ShareAlike 3.0 License, which allows others to remix, tweak, and build upon the work non-commercially, as long as the author is credited and the new creations are licensed under the identical terms

This article was originally published in a special issue, "Novel Therapeutic Approaches in Biomedicine and Pharmaceutical

Sciences" Indian J Pharm Sci 2021:83(6) Spl Issue "219-225" 